\title{
Bäcklund transformations for the Camassa-Holm equation
}

\author{
Alexander G. Rasin \\ Department of Mathematics, \\ Ariel University, Ariel, 40700, Israel \\ E-mail: rasin@ariel.ac.il \\ Jeremy Schiff \\ Department of Mathematics, \\ Bar-Ilan University, Ramat Gan, 52900, Israel \\ E-mail:schiff@math.biu.ac.il
}

October 21, 2018

\begin{abstract}
The Bäcklund transformation (BT) for the Camassa-Holm $(\mathrm{CH})$ equation is presented and discussed. Unlike the vast majority of BTs studied in the past, for $\mathrm{CH}$ the transformation acts on both the dependent and (one of) the independent variables. Superposition principles are given for the action of double BTs on the variables of the $\mathrm{CH}$ and the potential $\mathrm{CH}$ equations. Applications of the $\mathrm{BT}$ and its superposition principles are presented, specifically the construction of travelling wave solutions, a new method to construct multi-soliton, multi-cuspon and solitoncuspon solutions, and a derivation of generating functions for the local symmetries and conservation laws of the $\mathrm{CH}$ hierarchy.
\end{abstract}

\section{Introduction}

The original Bäcklund transformation (BT) arose in the context of differential geometry of surfaces in the 1880s [3]. In the modern era, BTs have been recognized as playing a central role in the theory of integrable differential equations [34, 59, 58, Their primary application is as a method to generate explicit solutions, exploiting the so-called superposition principle, an algebraic rule to "combine" two solutions obtained by BTs (from a given initial solution). However, in recent work [55] we have also shown how to derive local symmetries and conservation laws directly from BTs. There is also a deep relationship between BTs and the associated linear systems of integrable equations.

The Camassa-Holm $(\mathrm{CH})$ equation [9, 10] is by now recognized as one of the archetypes of integrable equations. It has (weak) "peakon" solutions - solitary waves with discontinuous first derivative at their crest - and numerous other types of travelling wave solution, including solitons (smooth solitary waves), cuspons and various periodic structures [40, 41, 7, 8, 33, 47, 48, 50, 37, 54. The integrability of the $\mathrm{CH}$ equation was already firmly 
established in [9], where a Lax pair and a bihamiltonian structure were given, and much further evidence for this has accumulated since then. There is an inverse scattering formalism [13, 14, explicit formulas can be found for multipeakon,multisoliton, multicuspon and soliton-cuspon solutions [60, 4, 5, 6, 22, 31, 39, 49, 18, 38, 43, 53, 44, 51, 52, 19, 63. there are an infinite number of local conservation laws [23, 56, 57, 28, 25, 27, 35, 11, 30, 24, and there is a rich algebra of symmetries [56, 57, 28, 25, 27, 24]. Other significant works on $\mathrm{CH}$ include studies of the stability of peakon and other exact solutions [15, 16, 17, 36] and interesting numerical studies [32, 45, 21, 12].

The aim of this paper is to fully explore the theory of the BT for the $\mathrm{CH}$ equation. In [60], one of us constructed a $\mathrm{BT}$ for the associated $\mathrm{CH}(\mathrm{aCH})$ equation, an equation related by a (field dependent) change of coordinates to the $\mathrm{CH}$ equation, and used this to construct some solutions of $\mathrm{CH}$ which could be regarded as superpositions of 2 travelling waves. However, this work was incomplete; an integration was required to reconstruct a solution of $\mathrm{CH}$ from a solution of aCH, which, in general, could not be done explicitly, severly limiting applicability. In the current paper we resolve this and other problems. The BT of CH differs from standard ones (for example, those of KdV and Sine-Gordon) in that it involves a transformation of both the dependent and one of the independent variables. However, remarkably, there is a nonlinear superposition principle for both of these transformations, which we develop and apply to the generation of multisoliton, multicuspon and soliton-cuspon solutions, as well as to the derivation of symmetries and conservation laws for $\mathrm{CH}$. The action of the BT on both dependent and independent variables is not unique to $\mathrm{CH}$; a similar situation exists for the Dym equation, which also exhibits nonanalytic solitons [62, 61].

The structure of this paper is as follows: In section 2 we recap the known results for the aCH equation. In section 3 we use them to derive the BT for $\mathrm{CH}$. Section 4 discusses the various forms of superposition principle. In section 5 we use the BT to obtain travelling wave solutions. The BT is used to construct soliton and cuspon solutions from which the standard peakon solutions can be obtained in a certain limit. Alas it does not seem to give a direct construction of peakons. However, various other unphysical solutions are also obtained. In section 6 we use the superposition principle to obtain cusponcuspon, soliton-soliton and cuspon-soliton solutions. In section 7, following [55], we use the BT to construct the conservation laws and symmetries of $\mathrm{CH}$. Section 8 contains some concluding remarks.

\section{Previous results}

The Camassa-Holm equation $(\mathrm{CH})$ [9] is

$$
m_{t}+2 u_{x} m+u m_{x}=0, \quad m=u-u_{x x},
$$

or equivalently

$$
u_{t}-u_{t x x}+3 u u_{x}-u u_{x x x}-2 u_{x} u_{x x}=0 .
$$

By translating $u$ and performing a Galilean transformation $x \rightarrow x-c t$ it is possible to introduce linear transport and linear dispersion terms into the equation, see for example [20]. All the results we present here can be generalized for the full class of equations considered in [20]. 
Writing $u=v_{x}$ and integrating once, we obtain the potential Camassa-Holm equation $(\mathrm{pCH})$

$$
v_{t}-v_{t x x}+\frac{3}{2} v_{x}^{2}-v_{x} v_{x x x}-\frac{1}{2} v_{x x}^{2}=0
$$

or, equivalently,

$$
n_{t}+\frac{1}{2} v_{x}^{2}+v_{x} n_{x}-\frac{1}{2}(v-n)^{2}=0, \quad n=v-v_{x x} .
$$

Evidently $n$ is a potential for $m, m=n_{x}$.

In [60] equation (11), under the assumption $m>0$, was transformed to the associated Camassa-Holm equation $(\mathrm{aCH})$

$$
2 p_{\tau}=-p^{2} u_{\xi}, \quad u=-\frac{p}{2}\left(\frac{p_{\tau}}{p}\right)_{\xi}+p^{2},
$$

with the help of transformation

$$
p=\sqrt{m}, \quad d \xi=\frac{1}{2} p d x-\frac{1}{2} p u d t, \quad \tau=t .
$$

This transformation implies

$$
\frac{\partial x}{\partial \xi}=\frac{2}{p}, \quad \frac{\partial x}{\partial \tau}=u, \quad \frac{\partial t}{\partial \xi}=0, \quad \frac{\partial t}{\partial \tau}=1 .
$$

A BT for aCH was found in [60]:

$$
p \rightarrow p-s_{\xi}, \quad u \rightarrow u+\frac{2 s_{\tau}}{p\left(p-s_{\xi}\right)},
$$

where $s$ satisfies

$$
\begin{aligned}
s_{\xi} & =-\frac{s^{2}}{p \alpha}+\frac{\alpha}{p}+p \\
s_{\tau} & =-s^{2}+\frac{p_{\tau}}{p} s+\alpha(\alpha+u) .
\end{aligned}
$$

The following nonlinear superposition principle was also given:

$$
p \rightarrow p-\left(\frac{(\alpha-\beta)\left(\alpha \beta-s_{\alpha} s_{\beta}\right)}{\beta s_{\alpha}-\alpha s_{\beta}}\right)_{\xi},
$$

where $s_{\alpha}, s_{\beta}$ are the solutions of (6]

In [55] the BT was used to find an infinite number of symmetries for aCH. These are given by the generating symmetry $X=Q^{p} \frac{\partial}{\partial p}+Q^{u} \frac{\partial}{\partial u}$ where

$$
Q^{p}=\frac{p\left(s_{\alpha}^{(1)}+s_{\alpha}^{(2)}\right)}{\alpha\left(s_{\alpha}^{(1)}-s_{\alpha}^{(2)}\right)}, \quad Q^{u}=-\frac{2 s_{\alpha}^{(1)}+2 s_{\alpha}^{(2)}+p u_{\xi}}{s_{\alpha}^{(1)}-s_{\alpha}^{(2)}} .
$$

Here $s_{\alpha}^{(1)}, s_{\alpha}^{(2)}$ are two different solutions of (6)]7]) for the same parameter $\alpha$. This symmetry depends upon $\alpha$; expansion in a (formal) power series in $\alpha$ gives the infinite hierarchy of symmetries. 


\section{The Bäcklund transformation for the Camassa-Holm equation}

In this section we obtain the $\mathrm{BT}$ for $\mathrm{CH}$ and $\mathrm{pCH}$ from the $\mathrm{BT}$ for aCH. With the help of (44) we write the BT (5),(6), (7) as

$$
u \rightarrow u-2 \alpha-\frac{2 \alpha\left(u_{x} s-\alpha u\right)}{s^{2}-\alpha^{2}},
$$

where $s$ satisfies

$$
\begin{aligned}
& s_{x}=-\frac{s^{2}}{2 \alpha}+\frac{1}{2}(m+\alpha), \\
& s_{t}=-s^{2}\left(1-\frac{u}{2 \alpha}\right)-u_{x} s+\frac{1}{2}\left(2 \alpha^{2}+\alpha u-u m\right) .
\end{aligned}
$$

This system for $s$ is equivalent to the Lax pair for CH. Note (12) can be simplified with the help of (11) and (11) to

$$
s_{t}=\alpha u_{x x}+2 \alpha s_{x}-u s_{x}-u_{x} s .
$$

In light of (4) the $\mathrm{BT}$ for $\mathrm{CH}$ must also involve the independent variable $x$. Using the first equation in (4), the change of the independent variable is

$$
\begin{aligned}
x_{\text {new }}-x & =\int\left(\frac{2}{p_{\text {new }}}-\frac{2}{p}\right) d \xi \\
& =\int\left(\frac{2}{p-s_{\xi}}-\frac{2}{p}\right) d \xi \\
& =\int \frac{2 s_{\xi}}{p\left(p-s_{\xi}\right)} d \xi \\
& =\int \frac{2 d s}{\frac{s^{2}}{\alpha}-\alpha} \\
& =\ln \left|\frac{s-\alpha}{s+\alpha}\right|+f(\tau) .
\end{aligned}
$$

In moving from the third to the fourth line here the formula for $s_{\xi}$ in (66) is used in the denominator but not in the numerator. The integration leaves undetermined an arbitrary function $f(\tau)$. Using the second equation in (4) it is straightforward to show this must be a constant, which can be taken, without loss of generality, to be zero. Thus the effect of the BT on the independent coordinates is

$$
x \rightarrow x+\ln \left|\frac{s-\alpha}{s+\alpha}\right|, \quad t \rightarrow t .
$$

There is no guarantee that this mapping will be a bijection. We will see later an example in which the BT generates several solutions out of one, in the case that this mapping is not 1 to 1 .

Using (5) and (6) it is straightforward to write down the BT for the field $p$

$$
p \rightarrow \frac{s^{2}-\alpha^{2}}{\alpha p}
$$


and hence also for the field $m=p^{2}=u-u_{x x}$

$$
m \rightarrow \frac{\left(s^{2}-\alpha^{2}\right)^{2}}{\alpha^{2} m} .
$$

Further calculations give the action of the BT for the pCH fields $v$ (satisfying $u=v_{x}$ ) and $n=v-v_{x x}$ :

$$
\begin{gathered}
n \rightarrow n-2 s, \\
v \rightarrow v+\frac{2 \alpha\left(\alpha u_{x}-u s\right)}{s^{2}-\alpha^{2}} .
\end{gathered}
$$

As mentioned above, the BT can be generalized for the full family of equations from [20]

$$
c_{1} u_{x}+c_{2} u_{x x x}+c_{3}\left(u_{t}+3 u u_{x}\right)=c_{4}\left(u_{t x x}+u u_{x x x}+2 u_{x} u_{x x}\right),
$$

where $c_{1}, c_{2}, c_{3}, c_{4}$ are constants. (This generalized equation is referred to in [26, 64] as the "CH-r equation".) The BT is

$$
x \rightarrow x+\sqrt{\frac{c_{4}}{c_{3}}} \ln \left|\frac{s \sqrt{c_{4}}-\alpha \sqrt{c_{3}}}{s \sqrt{c_{4}}+\alpha \sqrt{c_{3}}}\right|, \quad u \rightarrow u-2 \alpha-\frac{2 c_{3} \alpha^{2} u-2 c_{4} \alpha s u_{x}+c_{1} \alpha^{2}+c_{2} s^{2}}{c_{3} \alpha^{2}-c_{4} s^{2}} .
$$

Here $s$ satisfies

$$
\begin{aligned}
s_{x} & =-\frac{s^{2}}{2 \alpha}+\frac{\alpha}{2} \frac{2 c_{3} u-2 c_{4} u_{x x}+2 c_{3} \alpha+c_{1}}{2 c_{4} \alpha-c_{2}}, \\
s_{t} & =\alpha u_{x x}+2 \alpha s_{x}-u s_{x}-u_{x} s .
\end{aligned}
$$

Equation (19) includes the KdV, CH, and Hunter-Saxton (HS) 29] equations. The KdV equation can be obtained by putting $c_{1}=c_{4}=0$. The HS equation

$$
u_{t x}+\frac{1}{2} u_{x}^{2}+u u_{x x}=0
$$

can be obtained by putting $c_{1}=c_{2}=c_{3}=0$ and integrating with respect to $x$. The BT in this case is

$$
x \rightarrow x-\frac{2 \alpha}{s}, \quad u \rightarrow u-\frac{2 \alpha u_{x}}{s}-2 \alpha,
$$

where

$$
\begin{aligned}
& s_{x}=-\frac{s^{2}}{2 \alpha}-\frac{u_{x x}}{2}, \\
& s_{t}=\alpha u_{x x}+2 \alpha s_{x}-u s_{x}-u_{x} s .
\end{aligned}
$$

\section{The double Bäcklund transformation and super- position principles}

In this section we discuss double BTs for $\mathrm{CH}$ and $\mathrm{pCH}$. We also show the superposition principles for these equations.

As we saw in the previous section, a BT (which acts on the $\mathrm{CH}$ fields $u, m, p=\sqrt{m}$, the pCH fields $v, n$ and the independent coordinate $x$ according to equations (10), (16), (15), (18), (17), (14) respectively) is determined by a solution $s$ of (11), (12). We use 
the following notation: Denote by $s_{\alpha}, s_{\beta}$ etc the solutions of (11),(12) corresponding to parameters $\alpha, \beta$ etc. Denote the associated action on the fields by $u \rightarrow u_{\alpha}, m \rightarrow m_{\alpha}$ etc. Denote by $s_{\alpha \beta}$ the solution of (11), (12) with $u, m$ replaced by $u_{\alpha}, m_{\alpha}$ and parameter $\beta$ (i.e. we start with a solution obtained from a BT with parameter $\alpha$ and are now considering acting upon it by a further BT with parameter $\beta$ ). Denote the corresponding action on the fields by $u_{\alpha} \rightarrow u_{\alpha \beta}, m_{\alpha} \rightarrow m_{\alpha \beta}$ etc.

The fundamental fact about double BTs, as proved in [60], is that they commute, i.e. $u_{\alpha \beta}=u_{\beta \alpha}, m_{\alpha \beta}=m_{\beta \alpha}$ etc. From, for example, the transformation law for the pCH field $n$, (17), it immediately follows that

$$
s_{\alpha}+s_{\alpha \beta}=s_{\beta}+s_{\beta \alpha} .
$$

Checking the consitency of this with the versions of (11) and (12) satisfied by $s_{\alpha}, s_{\beta}, s_{\alpha \beta}, s_{\beta \alpha}$ we obtain

$$
s_{\alpha \beta}=-s_{\alpha}+\frac{(\alpha-\beta)\left(\alpha \beta-s_{\alpha} s_{\beta}\right)}{\beta s_{\alpha}-\alpha s_{\beta}}, \quad s_{\beta \alpha}=-s_{\beta}+\frac{(\alpha-\beta)\left(\alpha \beta-s_{\alpha} s_{\beta}\right)}{\beta s_{\alpha}-\alpha s_{\beta}} .
$$

In fact it is possible to check directly that these formulas for $s_{\alpha \beta}, s_{\beta \alpha}$ give solutions of the relevant versions of (11) and (12) without any need to assume (25).

From (26) it follows that once $s_{\alpha}$ and $s_{\beta}$ are known, it is possible to immediately find the action of a double BT. Using the transformation laws for $m, p, n, x$ and (26) we find

$$
\begin{aligned}
p_{\alpha \beta} & =\frac{\alpha \beta\left(\left(s_{\alpha}-s_{\beta}\right)^{2}-(\alpha-\beta)^{2}\right)}{\left(\beta s_{\alpha}-\alpha s_{\beta}\right)^{2}} p \\
m_{\alpha \beta} & =\frac{\alpha^{2} \beta^{2}\left(\left(s_{\alpha}-s_{\beta}\right)^{2}-(\alpha-\beta)^{2}\right)^{2}}{\left(\beta s_{\alpha}-\alpha s_{\beta}\right)^{4}} m \\
n_{\alpha \beta} & =n-2 \frac{(\alpha-\beta)\left(\alpha \beta-s_{\alpha} s_{\beta}\right)}{\beta s_{\alpha}-\alpha s_{\beta}} \\
x_{\alpha \beta} & =x+\ln \left|\frac{s_{\beta}-s_{\alpha}+\alpha-\beta}{s_{\beta}-s_{\alpha}-\alpha+\beta}\right|
\end{aligned}
$$

For $u$ and $v$ we proceed as follows. From (10) and (18) we obtain

$$
u_{\alpha}+u+\frac{1}{\alpha}\left(v_{\alpha}-v\right) s_{\alpha}=-2 \alpha
$$

and similarly

$$
\begin{aligned}
u_{\beta}+u+\frac{1}{\beta}\left(v_{\beta}-v\right) s_{\beta} & =-2 \beta, \\
u_{\alpha \beta}+u_{\beta}+\frac{1}{\alpha}\left(v_{\alpha \beta}-v_{\beta}\right) s_{\beta \alpha} & =-2 \alpha, \\
u_{\alpha \beta}+u_{\alpha}+\frac{1}{\beta}\left(v_{\alpha \beta}-v_{\alpha}\right) s_{\alpha \beta} & =-2 \beta .
\end{aligned}
$$

Eliminating $v_{\alpha}, v_{\beta}, v_{\alpha \beta}$ from these 4 relations, using (26) for $s_{\alpha \beta}$ and (10) for $u_{\alpha}, u_{\beta}$ we obtain

$$
u_{\alpha \beta}=u-\frac{2(\alpha-\beta)\left((\alpha-\beta)(\alpha+\beta+u)+\left(s_{\beta}-s_{\alpha}\right)\left(s_{\beta}+s_{\alpha}+u_{x}\right)\right)}{(\alpha-\beta)^{2}-\left(s_{\beta}-s_{\alpha}\right)^{2}} .
$$


Similarly, by first eliminating the fields $u$,

$$
v_{\alpha \beta}=v-\frac{2(\alpha-\beta)\left((\alpha-\beta) u_{x}+\left(s_{\beta}-s_{\alpha}\right) u+2\left(\alpha s_{\beta}-\beta s_{\alpha}\right)\right)}{(\alpha-\beta)^{2}-\left(s_{\beta}-s_{\alpha}\right)^{2}} .
$$

Equations (27), (28), (29), (230),(135) and (36) are algebraic formulas for the implementation of a double BT given $s_{\alpha}$ and $s_{\beta}$. However $s_{\alpha}$ and $s_{\beta}$ also determine the implementation of the original single BTs, so it is natural to try to eliminate them to obtain nonlinear superposition formulae for each of the quantities $p, m, n, x, u, v$. For example, for $x$ we have, from (14),

$$
s_{\alpha}=\frac{e^{x}+e^{x_{\alpha}}}{e^{x}-e^{x_{\alpha}}} \alpha, \quad s_{\beta}=\frac{e^{x}+e^{x_{\beta}}}{e^{x}-e^{x_{\beta}}} \beta
$$

and using these in (30) gives

$$
\frac{\left(e^{x}-e^{x_{\alpha}}\right)\left(e^{x_{\beta}}-e^{x_{\alpha \beta}}\right)}{\left(e^{x}-e^{x_{\beta}}\right)\left(e^{x_{\alpha}}-e^{x_{\alpha \beta}}\right)}=\frac{\alpha}{\beta} .
$$

Thus we see $e^{x}$ satisfies the cross-ratio equation, equation $\mathrm{A} 1[\delta=0]$ in the ABS classifciation [1]. Similarly for $n$ we obtain

$$
\beta\left(2 \alpha+n-n_{\alpha}\right)\left(2 \alpha-n_{\beta}+n_{\beta \alpha}\right)=\alpha\left(2 \beta+n-n_{\beta}\right)\left(2 \beta-n_{\alpha}+n_{\alpha \beta}\right),
$$

which is also the cross-ratio equation after a simple field redefintion. For $p$ the situation is a little more complicated as we have

$$
s_{\alpha}^{2}=\alpha\left(\alpha+p p_{\alpha}\right), \quad s_{\beta}^{2}=\beta\left(\beta+p p_{\beta}\right),
$$

and knowledge of $p_{\alpha}$ only determines $s_{\alpha}$ up to a sign. As a result, for given $p, p_{\alpha}, p_{\beta}$ there are 4 possibilities for $p_{\alpha \beta}$, which are given by solutions of the two multiquadratic quad-graph equations

$$
\begin{aligned}
4 \alpha \beta(\alpha-\beta)\left(p-p_{\alpha \beta}\right)\left(p_{\alpha}-p_{\beta}\right)+\alpha \beta\left(p-p_{\alpha \beta}\right)^{2}\left(p_{\alpha}-p_{\beta}\right)^{2} & \\
+\alpha(\alpha-\beta)\left(p p_{\alpha}-p_{\beta} p_{\alpha \beta}\right)^{2}+\beta(\beta-\alpha)\left(p p_{\beta}-p_{\alpha} p_{\alpha \beta}\right)^{2} & =0 \\
-4 \alpha \beta(\alpha+\beta)\left(p+p_{\alpha \beta}\right)\left(p_{\alpha}+p_{\beta}\right)-\alpha \beta\left(p+p_{\alpha \beta}\right)^{2}\left(p_{\alpha}+p_{\beta}\right)^{2} & \\
+\alpha(\alpha+\beta)\left(p p_{\alpha}-p_{\beta} p_{\alpha \beta}\right)^{2}+\beta(\beta+\alpha)\left(p p_{\beta}-p_{\alpha} p_{\alpha \beta}\right)^{2} & =0 .
\end{aligned}
$$

The first of these is precisely the $\mathrm{H}^{*}$ equation in the Atkinson-Nieszporski classification of integrable multiquadratic quad graph equations [2], as is the second after a simple field redefinition.

For $u$ and $v$ we have not succeeded to write a single superposition principle not involving any of the other fields. However, using the relations (31)-(34) it is possible to write the following superposition principles involving, respectively, just $u$ and $n$, and just $v$ and $n$ :

$$
\begin{aligned}
& \alpha\left(\frac{u_{\alpha}+u+2 \alpha}{n_{\alpha}-n}-\frac{u_{\alpha \beta}+u_{\beta}+2 \alpha}{n_{\alpha \beta}-n_{\beta}}\right)-\beta\left(\frac{u_{\beta}+u+2 \beta}{n_{\beta}-n}-\frac{u_{\alpha \beta}+u_{\alpha}+2 \beta}{n_{\alpha \beta}-n_{\alpha}}\right)=0 \\
& \frac{\left(v_{\beta}-v\right)\left(n_{\beta}-n\right)+\left(v_{\alpha \beta}-v_{\alpha}\right)\left(n_{\alpha \beta}-n_{\alpha}\right)}{\beta}-\frac{\left(v_{\alpha}-v\right)\left(n_{\alpha}-n\right)+\left(v_{\alpha \beta}-v_{\beta}\right)\left(n_{\alpha \beta}-n_{\beta}\right)}{\alpha} \\
= & 8(\beta-\alpha) .
\end{aligned}
$$

Here the fields $n$ satisfy the cross-ratio type equation (38). 


\section{Travelling wave solutions}

In this section we apply the BT (10),(14) where $s$ satisfies (11), (12) to the constant solution of $\mathrm{CH} u=u_{0} \neq 0$, to obtain travelling wave solutions, specifically soliton and cuspon solutions. These and other travelling wave solutions have been extensively studied in the literature, see for example [40, 41, 7, 8, 33, 47, 48, 50, 37, 54], and the BT is just one of many methods to derive them. The advantages of the BT will become apparent when we study superposition in the next section.

If $\alpha\left(\alpha+u_{0}\right)>0$ there are two kinds of real solutions of (111),(12):

$$
s_{\alpha}=\sqrt{\alpha\left(u_{0}+\alpha\right)} \tanh \left(\frac{\sqrt{\alpha\left(u_{0}+\alpha\right)}\left(x-x_{0}+\left(2 \alpha-u_{0}\right) t\right)}{2 \alpha}\right),
$$

which we call the "tanh-type" solution, and the same with tanh replaced by coth, which we call the "coth-type" solution. As we will see both of these give rise to travelling wave solutions. If $\alpha\left(\alpha+u_{0}\right)<0$ then there are real solutions

$$
s_{\alpha}=\sqrt{-\alpha\left(u_{0}+\alpha\right)} \tan \left(\frac{\sqrt{-\alpha\left(u_{0}+\alpha\right)}\left(x-x_{0}+\left(2 \alpha-u_{0}\right) t\right)}{2 \alpha}\right),
$$

and the same with tan replaced by cot, and an overall minus sign. Both of these give rise to periodic solutions (see for example [7, 37]), but these will not be studied here.

Returning to the case $\alpha\left(\alpha+u_{0}\right)>0$, it is useful to write $\alpha+u_{0}=\alpha U^{2}$, where $U>0$, so the solution (43) becomes

$$
s_{\alpha}=\alpha U \tanh \left(\frac{U}{2}\left(x-x_{0}+\left(3-U^{2}\right) \alpha t\right)\right),
$$

and the same with coth for a coth-type solution. Using (10), (14) the resulting solution is $u_{\alpha}\left(x_{\alpha}, t\right)$ where

$$
\begin{aligned}
u_{\alpha} & =\alpha\left(U^{2}-3\right)+\frac{2 \alpha\left(U^{2}-1\right)}{U^{2} \tanh ^{2}\left(\frac{U}{2}\left(x-x_{0}+\left(3-U^{2}\right) \alpha t\right)\right)-1}, \\
x_{\alpha} & =x+\ln \left|\frac{U \tanh \left(\frac{U}{2}\left(x-x_{0}+\left(3-U^{2}\right) \alpha t\right)\right)-1}{U \tanh \left(\frac{U}{2}\left(x-x_{0}+\left(3-U^{2}\right) \alpha t\right)\right)+1}\right|
\end{aligned}
$$

or the same with coth. Finally, writing $z=x-x_{0}+\left(3-U^{2}\right) \alpha$, the solution becomes $u_{\alpha}\left(x_{\alpha}, t\right)$ where

$$
\begin{aligned}
u_{\alpha} & =\alpha\left(U^{2}-3\right)+\frac{2 \alpha\left(U^{2}-1\right)}{U^{2} \tanh ^{2} \frac{1}{2} U z-1}, \\
x_{\alpha}-x_{0}+\left(3-U^{2}\right) \alpha t & =z+\ln \left|\frac{U \tanh \frac{1}{2} U z-1}{U \tanh \frac{1}{2} U z+1}\right|,
\end{aligned}
$$

this being a tanh-type solution, or a coth-type solution, which is the same with tanh replaced by coth. Both tanh-type and coth-type solutions are travelling waves with speed $c=\left(U^{2}-3\right) \alpha$, written in an implicit form. The first step in analyzing these solutions is to decide whether the maps from $x_{\alpha}$ to $z$ are bijections. For tanh-type solutions with $U<1$, neither the factor in the numerator or in the denominator inside the ln can vanish, and 
thus $x_{\alpha}$ only tends to (plus or minus) infinity as $z$ tends to (plus or minus) infinity. The corresponding solutions are solitons which tend to $u_{0}=\alpha\left(U^{2}-1\right)$ at spatial infinity, with speed $c=\alpha\left(U^{2}-3\right)$ and central elevation $-\alpha\left(1+U^{2}\right)=c-2 u_{0}$. Note that since

$$
U=\sqrt{\frac{3 u_{0}-c}{u_{0}-c}}
$$

and $0<U<1$ we must either have $c<3 u_{0}<0$ or $0<3 u_{0}<c$. Figure 1 displays the soliton profile for $c=2$ and $u_{0}=0.5,0.1,0.02$. (For negative $u_{0}$ and $c$ the soliton is inverted.)

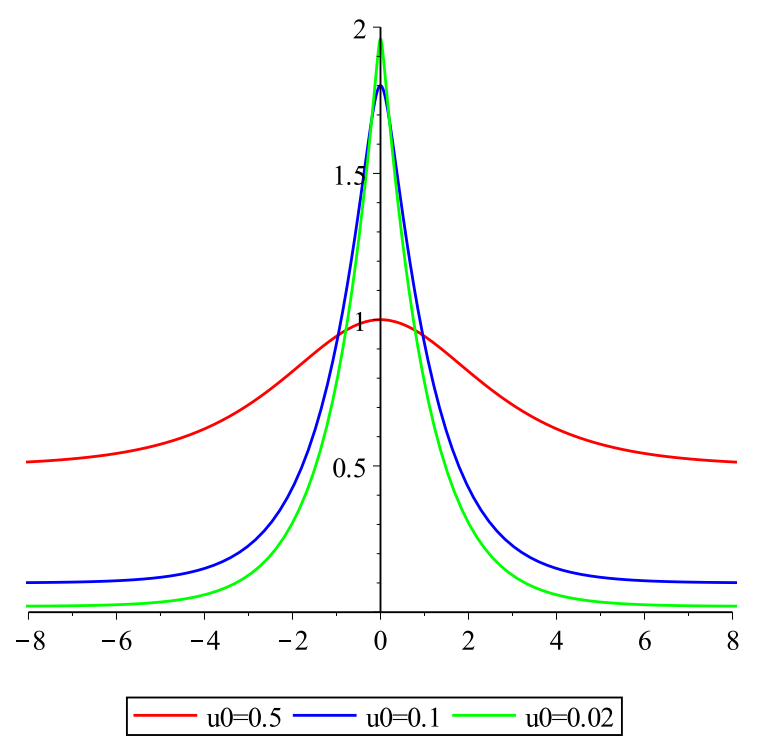

Figure 1: Soliton profile, $c=2, u_{0}=0.5,0.1,0.02$.

Of particular interest is the limit of the soliton for fixed $c$ and $u_{0} \downarrow 0$ (for $c>0$ ) or $u_{0} \uparrow 0$ (for $c<0$ ). Figure 2 shows $x_{\alpha}$ as a function of $z$ (for $x_{0}=t=0$ ), $u_{\alpha}$ as a function of $z$ and $u_{\alpha}$ as a function of $x_{\alpha}$ in the case $c=2, u_{0}=10^{-8} \cdot x_{\alpha}$ is close to zero, and $u_{\alpha}$ is close to $c$ for a large interval of $z$ values of size $O\left(\left|\ln \left(u_{0} / c\right)\right|\right)$ around $z=0$. In the plot of $u_{\alpha}$ against $x_{\alpha}$ this gives rise to a sharp peak. This is the peakon limit. To see this analytically it is possible to use (48) to find $z$ in terms of $u$ (with a \pm uncertainty as it is necessary to take a square root), and then (49) becomes

$$
\begin{aligned}
x_{\alpha}-x_{0}+\left(3-U^{2}\right) \alpha t= & \pm\left(2 \sqrt{\frac{c-u_{0}}{c-3 u_{0}}} \operatorname{arctanh}\left(\sqrt{\frac{c-u_{0}}{c-3 u_{0}}} \sqrt{1-\frac{2 u_{0}}{c-u_{\alpha}}}\right)\right. \\
& \left.+\ln \left(\frac{c-u_{\alpha}}{2 u_{0}}\left(1-\sqrt{1-\frac{2 u_{0}}{c-u_{\alpha}}}\right)^{2}\right)\right) .
\end{aligned}
$$

Both terms on the RHS diverge as $u_{0} \rightarrow 0$, but it is straightforward to extract the divergent behavior, which cancels between the terms, and to obtain the limit, which is simply $\pm \ln \left(\frac{u_{\alpha}}{c}\right)$.

Moving now to tanh-type solutions with $U>1$, from (49) we expect $x_{\alpha}$ to diverge when $\tanh \frac{1}{2} U z= \pm \overline{\frac{1}{U} \text { and thus the map from } z \text { to }} x_{\alpha}$ will not be a bijection. Figure 3 shows $x_{\alpha}$ and $u_{\alpha}$ as functions of $z$ for $c=2$ and $u_{0}=3$. The map from $z$ to $x_{\alpha}$ is 3 to 

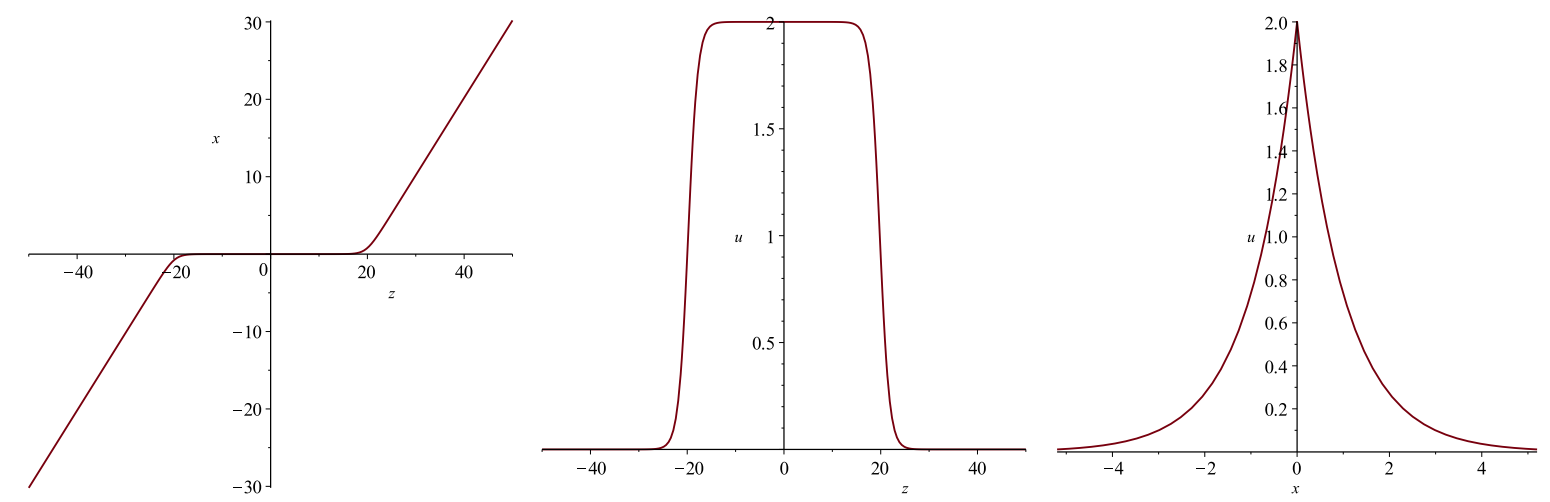

Figure 2: The soliton with $c=2$, approaching the peakon limit. $u_{0}=10^{-8} . x$ as a function of $z, u$ as a function of $z$ and $u$ as a function of $x$.
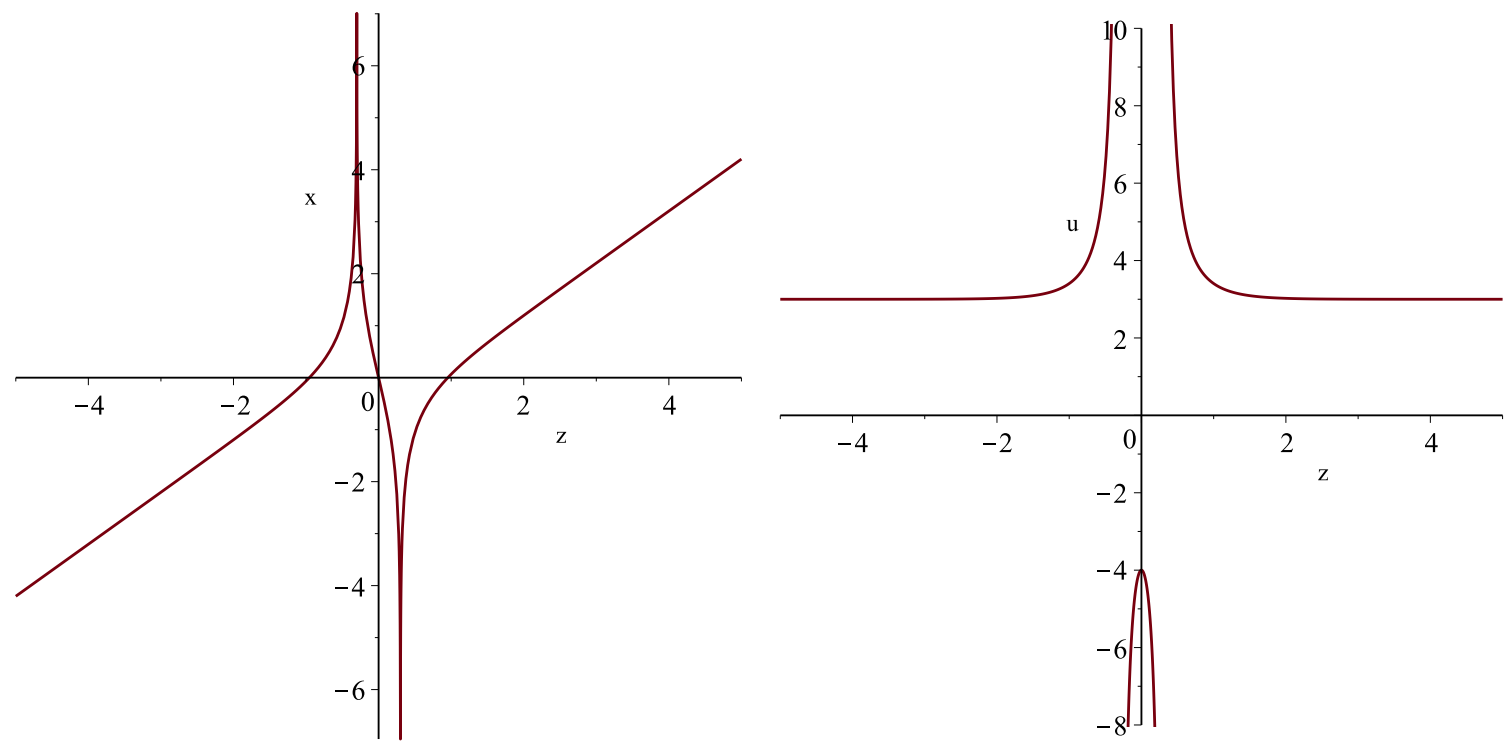

Figure 3: tanh-type solutions with $c=2$ and $u_{0}=3(U=\sqrt{7}>1), x_{\alpha}$ and $u_{\alpha}$ as functions of $z$. The map from $z$ to $x_{\alpha}$ is not $1-1$.

1 and thus there are 3 corresponding solutions of $\mathrm{CH}$, depicted in Figure 4. Since these are all unbounded we do not devote further attention to them.

Moving now to coth-type solutions, the situation is very similar, but now the map from $z$ to $x_{\alpha}$ will be $1-1$ if $U>1$ and many to 1 if $U<1$, and there is a subtlety arising due to the divergence of $\operatorname{coth} \frac{1}{2} U z$ at $z=0$. For coth-type solutions with $U<1$, $x_{\alpha}$ diverges when $\operatorname{coth} \frac{1}{2} U z= \pm \frac{1}{U}$. The map from $z$ to $\overline{x_{\alpha} \text { is once again } 3 \text { to } 1 \text {. Figure }}$ 5 shows $x_{\alpha}$ and $u_{\alpha}$ as functions of $z$ for $c=2$ and $u_{0}=\frac{1}{2}$, and Figure 6 shows the 3 corresponding solutions of $\mathrm{CH}$. The subtlety, as can be seen in Figure 7, is that the solution corresponding to the range of $z$ 's that includes zero, has a cusp at $z=0$, arising from the divergence of $\operatorname{coth} \frac{1}{2} U z$. Since at this point $u_{\alpha}$ is not differentiable, it is necessary to ask in what sense this is a solution of $\mathrm{CH}$. Fortunately, the value of $u_{\alpha}$ at the cusp is $c$, which makes it possible to interpret the solution in a weak sense [37], though we do not go into details here. 

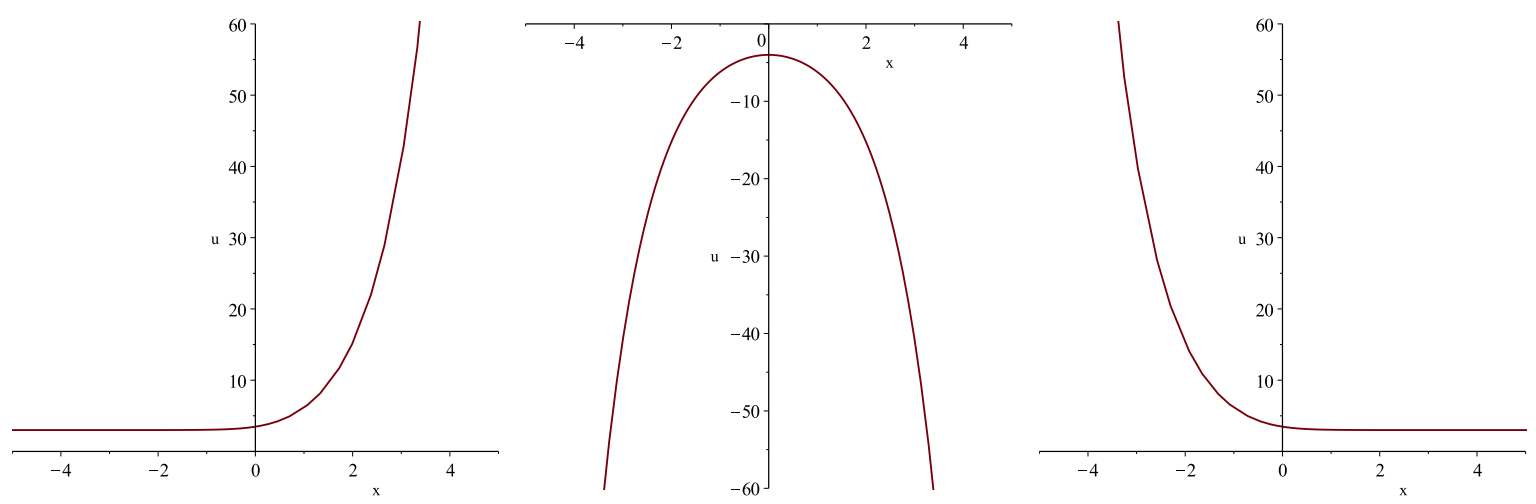

Figure 4: tanh-type solutions with $c=2$ and $u_{o}=3(U=\sqrt{7}>1), u_{\alpha}$ as a function of $x_{\alpha}$ for the 3 unbounded solutions of $\mathrm{CH}$.
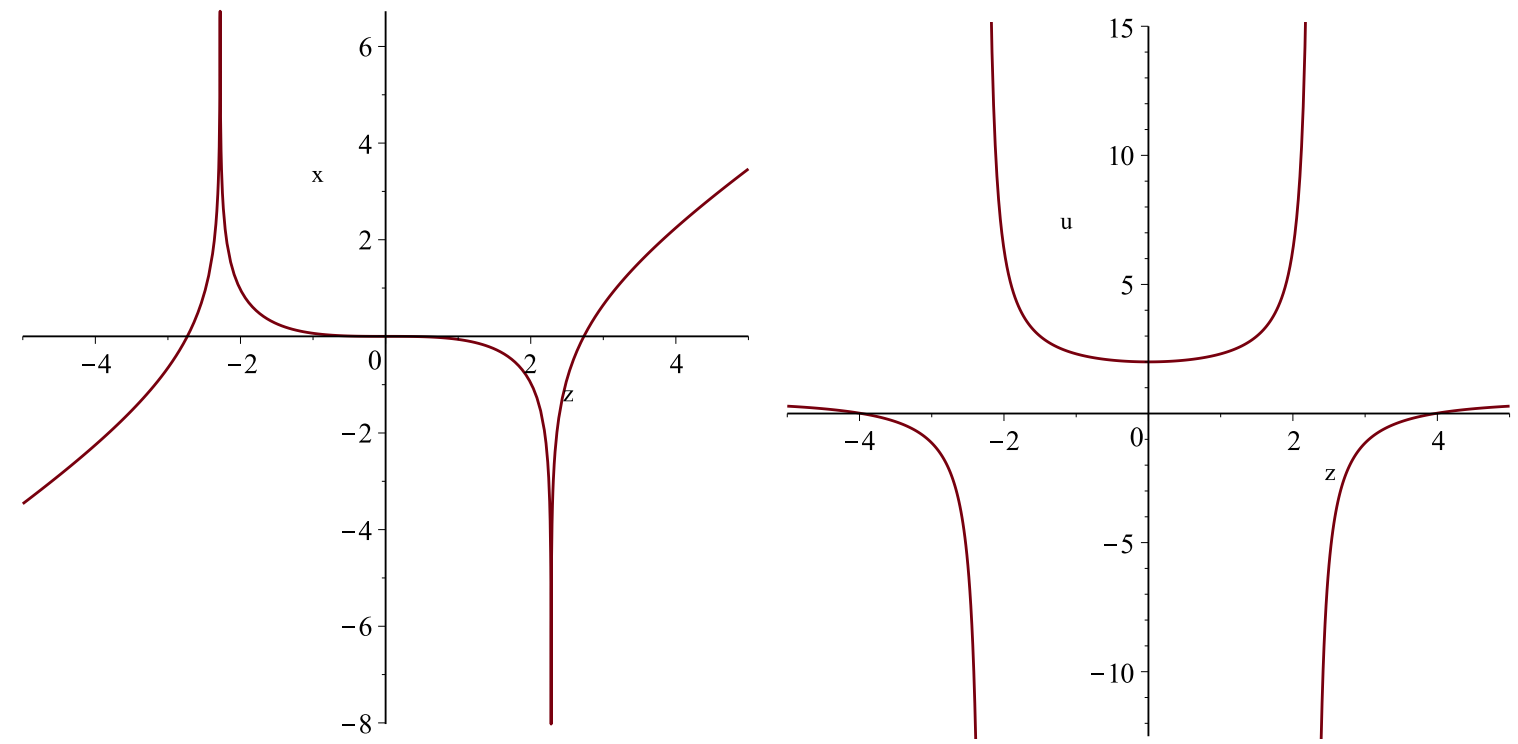

Figure 5: coth-type solutions with $c=2$ and $u_{0}=\frac{1}{2}\left(U=\frac{1}{\sqrt{3}}<1\right), x_{\alpha}$ and $u_{\alpha}$ as functions of $z$. The map from $z$ to $x_{\alpha}$ is not $1-1$.

For coth-type solutions with $U>1$, the map from $z$ to $x_{\alpha}$ is a bijection, and once again there is a single solution of $\mathrm{CH}$, but with a cusp at $z=0$ - this is the cuspon solution. Due to the requirement $U>1$ cuspon solutions only exist with speed $c<u_{0}$ if $u_{0}$ is positive, and speed $c>u_{0}$ if $u_{0}$ is negative. Figure 8 illustrates cuspon solutions with $c=2$ for $u_{0}=-1,-0.5,-0.1$. (For positive $u_{0}$ the cuspon is inverted.) Note that the central elevation of the cusp is $c$, as required for it to be a weak solution. For $c>0$ $(c<0)$ it is possible to consider the limit of the cuspon as $u_{0} \uparrow 0\left(u_{0} \downarrow 0\right)$, and this is once again the peakon limit. 

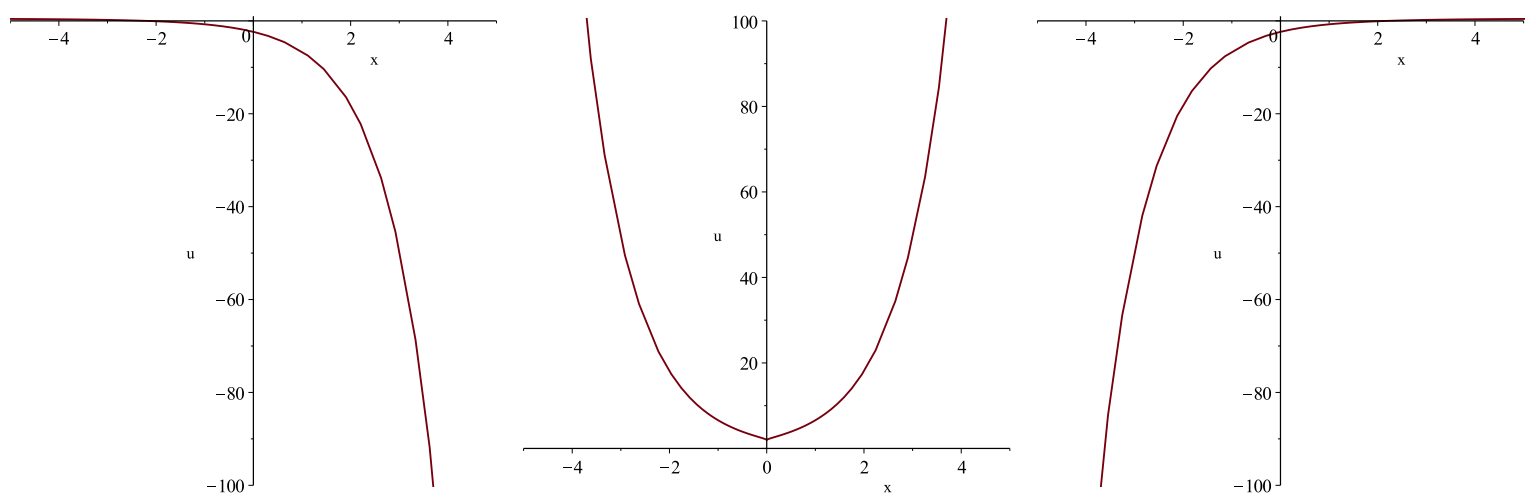

Figure 6: coth-type solutions with $c=2$ and $u_{0}=\frac{1}{2}\left(U=\frac{1}{\sqrt{3}}<1\right), u_{\alpha}$ as a function of $x_{\alpha}$ for the 3 unbounded solutions of $\mathrm{CH}$.

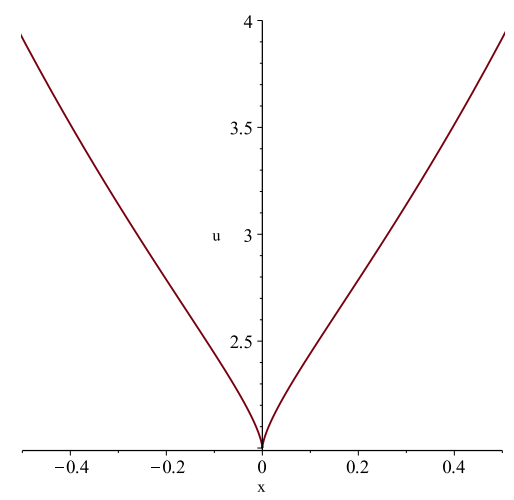

Figure 7: coth-type solutions with $c=2$ and $u_{0}=\frac{1}{2}\left(U=\frac{1}{\sqrt{3}}<1\right)$, close up on the cusp in one of the solutions of $\mathrm{CH}$.

We summarize the travelling waves presented in this section in the following table. All the solutions have asymptotic height $u_{0}$ :

\begin{tabular}{|c|c|c|c|}
\hline tanh-type & $U>1$ & $\begin{array}{l}\text { soliton } \\
\text { unphysical }\end{array}$ & $\begin{array}{l}\text { central elevation } c-2 u_{0} \\
u_{0}>0: c>3 u_{0} \\
u_{0}<0: c<3 u_{0} \text { inverted }\end{array}$ \\
\hline coth-type & $\begin{array}{l}U<1 \\
U<1\end{array}$ & $\begin{array}{l}\text { unphysical } \\
\text { cuspon }\end{array}$ & $\begin{array}{l}\text { central elevation } c \\
u_{0}>0: c<u_{0} \text { inverted } \\
u_{0}<0: c>u_{0}\end{array}$ \\
\hline
\end{tabular}




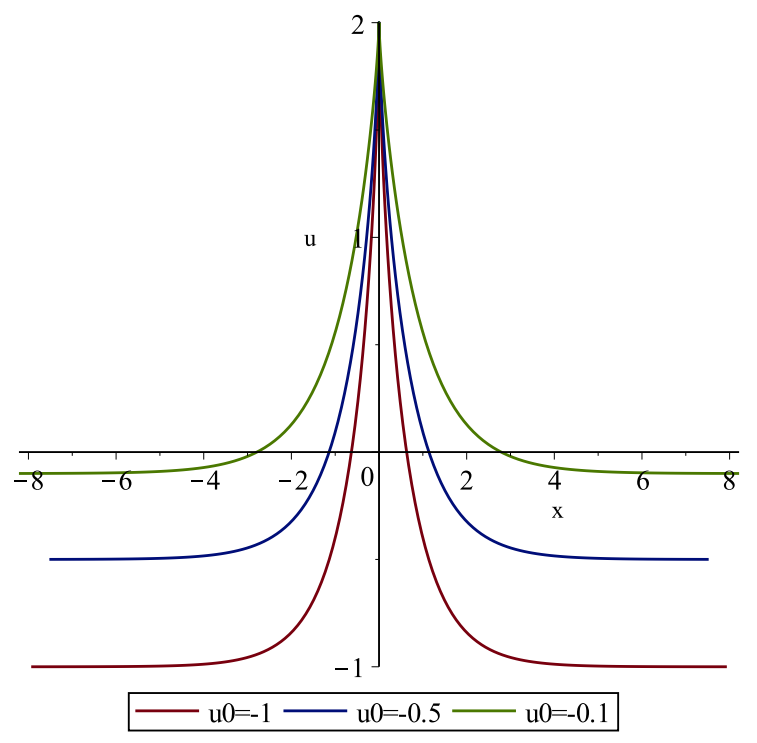

Figure 8: Cuspon profile, $c=2, u_{0}=-1,-0.5,-0.1$.

\section{Two wave solutions}

The first investigations of two wave solutions were [60] and [22], both of which required some element of numerical computation. However, since then, a substantial literature [31, 39, 49, 18, 38, 43, 53, 44, 52, 19, 63, has developed on multisoliton, multicuspon and soliton-cuspon solutions. The known methods for analytic construction of solutions include a determinantal formula based on the inverse scattering approach, a Hirota bilinear form for $\mathrm{CH}$ and a reciprocal transformation relating the $\mathrm{CH}$ hierarchy to the $\mathrm{KdV}$ hierarchy. (For multipeakon solutions very different techniques are involved [9, 4, 6, 5, 51].) As we will shortly see, use of the superposition principle gives a further very simple method.

In our approach, two wave solutions should be obtained using formulas (35) and (30), taking $u=u_{0}$ to be constant and $s_{\alpha}\left(s_{\beta}\right)$ either of tanh-type, as given in (45) where $U=U_{\alpha}=\sqrt{1+\frac{u_{0}}{\alpha}}\left(U=U_{\beta}=\sqrt{1+\frac{u_{0}}{\beta}}\right)$ and $x_{0}=x_{\alpha, 0}\left(x_{0}=x_{\beta, 0}\right)$, or of coth-type, which is identical but with coth. The only question is which superpositions of this type give maps from $x$ to $x_{\alpha \beta}$ that are 1-1.

Proposition. The following 3 superpositions give maps from $x$ to $x_{\alpha \beta}$ which are 1-1:

1. tanh-type solutions $s_{\alpha}$ with $U_{\alpha}<1$ with tanh-type solutions $s_{\beta}$ with $U_{\beta}>1$ (so $\left.\frac{u_{0}}{\alpha}<0<\frac{u_{0}}{\beta}\right)$ - soliton-cuspon superpositions.

2. tanh-type solutions $s_{\alpha}$ with $U_{\alpha}<1$ with coth-type solutions $s_{\beta}$ with $U_{\beta}<1$, with $U_{\alpha}<U_{\beta}-$ soliton-soliton superpositions.

3. tanh-type solutions $s_{\alpha}$ with $U_{\alpha}>1$ with coth-type solutions $s_{\beta}$ with $U_{\beta}>1$, with $U_{\beta}<U_{\alpha}$ - cuspon-cuspon superpositions.

Note here, for example, that a soliton-soliton superposition is not as we might expect, the superposition of two tanh-type solutions with $U<1$, but the superposition of a tanh-type solution with $U<1$ with a unphysical coth-type solution with $U<1$. 
Proof. It is necessary to show in each case that neither the numerator or denominator of the expression inside the $\ln$ in (30) vanishes, i.e. that $\left|s_{\beta}-s_{\alpha}\right| \neq|\beta-\alpha|$. In the calculations below we repeatedly use the identities

$$
\alpha=\frac{u_{0}}{U_{\alpha}^{2}-1}, \quad \beta=\frac{u_{0}}{U_{\beta}^{2}-1} .
$$

1. In this case we have

$$
\begin{aligned}
\left|s_{\beta}-s_{\alpha}\right| & =\left|\beta U_{\beta} \tanh (\ldots)-\alpha U_{\alpha} \tanh (\ldots)\right| \\
& <\left|\beta U_{\beta}\right|+\left|\alpha U_{\alpha}\right| \quad \text { as }|\tanh |<1 \\
& =\left|\frac{u_{0} U_{\beta}}{U_{\beta}^{2}-1}\right|+\left|\frac{u_{0} U_{\alpha}}{U_{\alpha}^{2}-1}\right| \\
& =\left|u_{0}\right|\left(\frac{U_{\beta}}{U_{\beta}^{2}-1}-\frac{U_{\alpha}}{U_{\alpha}^{2}-1}\right) \quad \text { as } 0<U_{\alpha}<1<U_{\beta} \\
& =\left|u_{0}\right|\left(\frac{1}{U_{\beta}^{2}-1}+\frac{1}{U_{\beta}+1}-\frac{1}{U_{\alpha}^{2}-1}-\frac{1}{U_{\alpha}+1}\right) \\
& <\left|u_{0}\right|\left(\frac{1}{U_{\beta}^{2}-1}-\frac{1}{U_{\alpha}^{2}-1}\right) \quad \text { as } \frac{1}{U_{\alpha}+1}>\frac{1}{U_{\beta}+1} \\
& =|\beta-\alpha| .
\end{aligned}
$$

2. In this case we have

$$
\begin{aligned}
\left|s_{\alpha}\right|+|\beta-\alpha| & =\left|\alpha U_{\alpha} \tanh (\ldots)\right|+|\beta-\alpha| \\
& <\left|\alpha U_{\alpha}\right|+|\beta-\alpha| \text { as }|\tanh |<1 \\
& =\left|\frac{u_{0} U_{\alpha}}{U_{\alpha}^{2}-1}\right|+\left|\frac{u_{0}}{U_{\beta}^{2}-1}-\frac{u_{0}}{U_{\alpha}^{2}-1}\right| \\
& =\left|u_{0}\right|\left(\frac{U_{\alpha}}{1-U_{\alpha}^{2}}+\frac{1}{1-U_{\beta}^{2}}-\frac{1}{1-U_{\alpha}^{2}}\right) \quad \text { as } 0<U_{\alpha}<U_{\beta}<1 \\
& =\left|u_{0}\right|\left(\frac{1}{1-U_{\beta}^{2}}-\frac{1}{1+U_{\alpha}}\right) \\
& =\left|u_{0}\right|\left(\frac{U_{\beta}}{1-U_{\beta}^{2}}+\frac{1}{1+U_{\beta}}-\frac{1}{1+U_{\alpha}}\right) \\
& <\left|u_{0}\right| \frac{U_{\beta}}{1-U_{\beta}^{2}} \text { as } \frac{1}{1+U_{\beta}}<\frac{1}{1+U_{\alpha}} \\
& =\left|\beta U_{\beta}\right| \\
& <\left|\beta U_{\beta} \operatorname{coth}(\ldots)\right| \text { as }|\operatorname{coth}|>1 \\
& =\left|s_{\beta}\right|
\end{aligned}
$$

This contradicts $\left|s_{\beta}-s_{\alpha}\right|=|\beta-\alpha|$, as the latter implies $\left|s_{\beta}\right| \leq\left|s_{\alpha}\right|+|\beta-\alpha|$. 
3. Similarly to case 2 we have

$$
\begin{aligned}
\left|s_{\alpha}\right|+|\beta-\alpha| & =\left|\alpha U_{\alpha} \tanh (\ldots)\right|+|\beta-\alpha| \\
& <\left|\alpha U_{\alpha}\right|+|\beta-\alpha| \text { as }|\tanh |<1 \\
& =\left|\frac{u_{0} U_{\alpha}}{U_{\alpha}^{2}-1}\right|+\left|\frac{u_{0}}{U_{\beta}^{2}-1}-\frac{u_{0}}{U_{\alpha}^{2}-1}\right| \\
& =\left|u_{0}\right|\left(\frac{U_{\alpha}}{U_{\alpha}^{2}-1}+\frac{1}{U_{\beta}^{2}-1}-\frac{1}{U_{\alpha}^{2}-1}\right) \quad \text { as } 1<U_{\beta}<U_{\alpha} \\
& =\left|u_{0}\right|\left(\frac{1}{U_{\beta}^{2}-1}+\frac{1}{U_{\alpha}+1}\right) \\
& =\left|u_{0}\right|\left(\frac{U_{\beta}}{U_{\beta}^{2}-1}-\frac{1}{U_{\beta}+1}+\frac{1}{U_{\alpha}+1}\right) \\
& <\left|u_{0}\right| \frac{U_{\beta}}{U_{\beta}^{2}-1} \text { as } \frac{1}{U_{\alpha}+1}<\frac{1}{U_{\beta}+1} \\
& =\left|\beta U_{\beta}\right| \\
& <\left|\beta U_{\beta} \operatorname{coth}(\ldots)\right| \text { as }|\operatorname{coth}|>1 \\
& =\left|s_{\beta}\right|
\end{aligned}
$$

It remains to present the plots of some superpositions. Figure 9 shows a tanh-tanh superposition with $u_{0}=1, x_{\alpha, 0}=0, x_{\beta, 0}=2, c_{\alpha}=u_{0}-2 \alpha=4, c_{\beta}=u_{0}-2 \beta=-1$. (For $u_{0}>0$ such solutions exist provded $c_{\alpha}>3 u_{0}$ and $c_{\beta}<u_{0}$ - note here that $c_{\beta}$ can be positive or negative.) Figure 10 shows a tanh-coth soliton-soliton superposition with $u_{0}=1, x_{\alpha, 0}=0, x_{\beta, 0}=-10, c_{\alpha}=u_{0}-2 \alpha=4, c_{\beta}=u_{0}-2 \beta=6$. Figure 11 shows a tanh-coth cuspon-cuspon superposition with $u_{0}=1, x_{\alpha, 0}=0, x_{\beta, 0}=-2$, $c_{\alpha}=u_{0}-2 \alpha=-1, c_{\beta}=u_{0}-2 \beta=-2$.
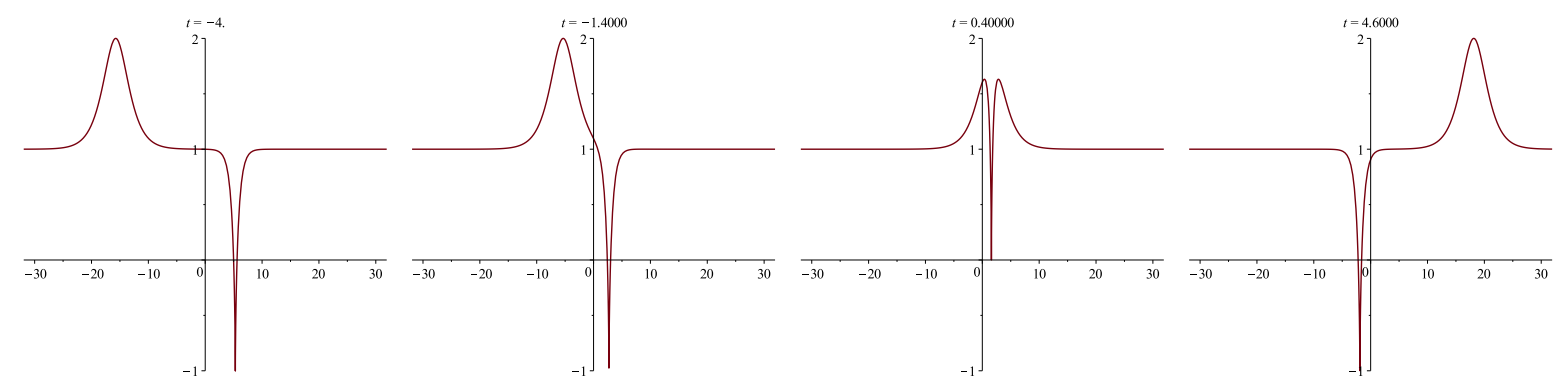

Figure 9: Tanh-Tanh Superposition. $u_{0}=1, x_{\alpha, 0}=0, x_{\beta, 0}=2, c_{\alpha}=4, c_{\beta}=-1$. Plots for times $t=-4,-1.4,0.4,4.6$ from left to right.

Note that in all the plots we have taken $u_{0}>0$, in which case, as in the previous section, the soliton solutions have positive speed $c>3 u_{0}$ and central elevation $c-2 u_{0}>$ $u_{0}$, whereas the cuspon solutions have speed $c<u_{0}$, which can be positive or negative, and central elevation $c<u_{0}$ (i.e. they might be called "anticuspons"). In the case $u_{0}<0$ everything is inverted and reversed (reflecting the $t \rightarrow-t, u \rightarrow-u$ symmetry of (2): solitons have negative speed $c<3 u_{0}$ and central elevation $c-2 u_{0}<u_{0}$ (antisolitons), and cuspons have speed $c>u_{0}$ and central elevation $c>u_{0}$. 

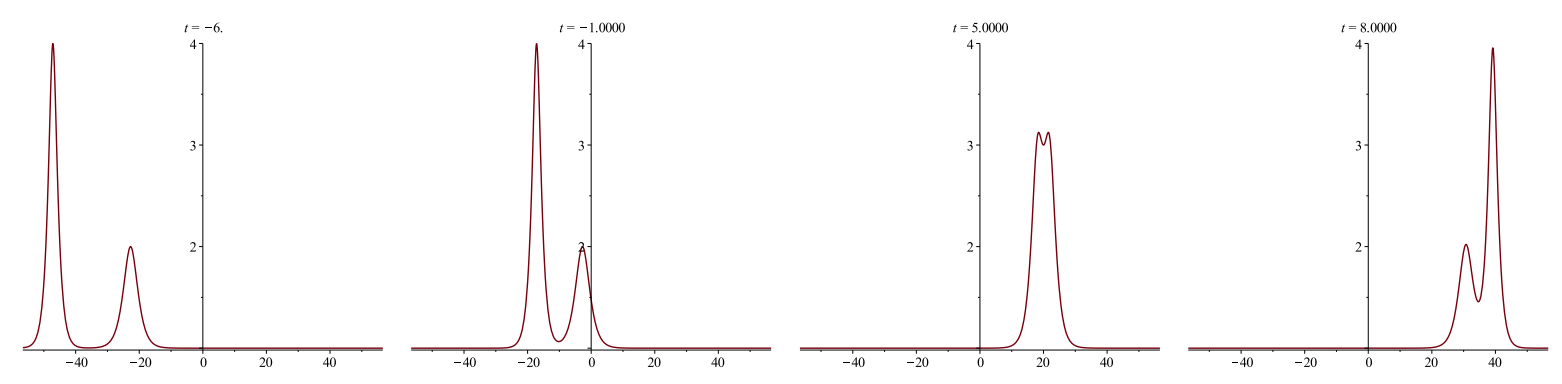

Figure 10: Tanh-Coth Soliton-Soliton Superposition. $u_{0}=1, x_{\alpha, 0}=0, x_{\beta, 0}=-10$, $c_{\alpha}=4, c_{\beta}=6$. Plots for times $t=-6,-1,5,8$ from left to right.
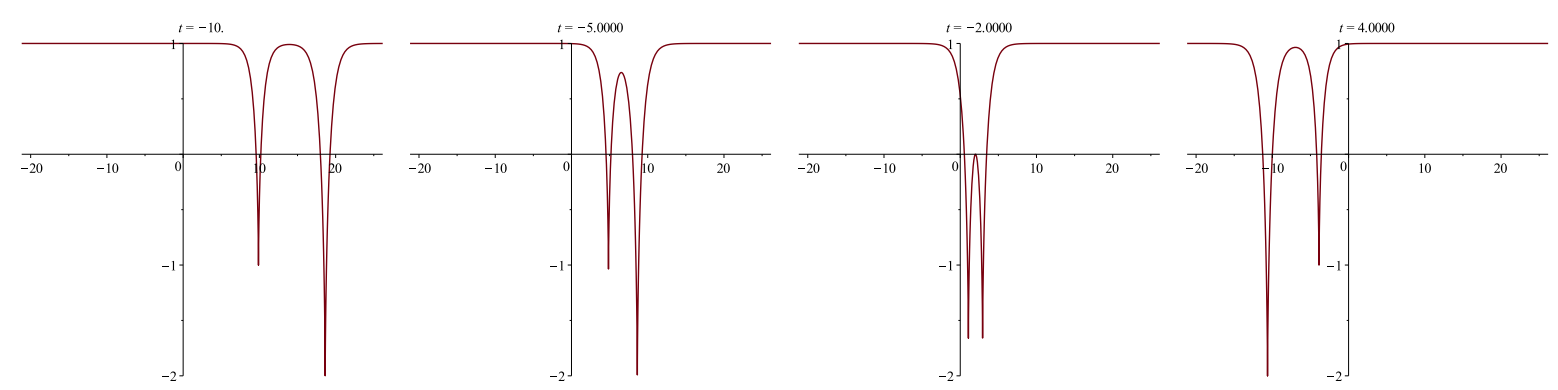

Figure 11: Tanh-Coth Cuspon-Cuspon Superposition. $u_{0}=1, x_{\alpha, 0}=0, x_{\beta, 0}=-2$, $c_{\alpha}=-1, c_{\beta}=-2$. Plots for times $t=-10,-5,-2,4$ from left to right.

\section{Symmetries and conservation laws for the Camassa- Holm equation}

In this section we show how to use the BT to obtain infinite hierarchies of symmetries and conservation laws for $\mathrm{CH}$ and $\mathrm{pCH}$, following the general methodology described in [55. The discussion of symmetries of $\mathrm{CH}$ in the literature is limited, though the existence of an infinite number of symmetries is implicit from the bihamiltonian structure given in [9]. In the series of papers [56, 57, 28, 25, 27, Reyes and collaborators present nonlocal symmetries of $\mathrm{CH}$ depending on a parameter, and then expand in powers of the parameter to obtain local symmetries, though limited details are given. Some explicit formulae appear in [24]. Our approach is related, but we will not discuss the connection explicitly.

As a starting point for our discussion of symmetries we could take the generating symmetry (9) for aCH, and work out the induced action on $x$, the independent variable in $\mathrm{CH}$. But a more direct approach is to look at the superposition principle (35), (30) in the limit that $\beta$ tends to $\alpha$, but $s_{\beta}$ tends to a second solution of (11)-(12) distinct from $s_{\alpha}$. More explicity, setting $\beta=\alpha-\frac{1}{2} \epsilon, s_{\alpha}=s_{\alpha}^{(1)}, s_{\beta}=s_{\alpha}^{(2)}+O(\epsilon)$ in (35), (30) we obtain

$$
\begin{aligned}
& u_{\alpha, \alpha-\frac{1}{2} \epsilon}=u+\epsilon \frac{s_{\alpha}^{(1)}+s_{\alpha}^{(2)}+u_{x}}{s_{\alpha}^{(2)}-s_{\alpha}^{(1)}}+O\left(\epsilon^{2}\right), \\
& x_{\alpha, \alpha-\frac{1}{2} \epsilon}=x+\frac{\epsilon}{s_{\alpha}^{(2)}-s_{\alpha}^{(1)}}+O\left(\epsilon^{2}\right) .
\end{aligned}
$$


We deduce the generating symmetry for $\mathrm{CH} X=Q^{x} \frac{\partial}{\partial x}+Q^{u} \frac{\partial}{\partial u}$ where

$$
Q^{x}=\frac{1}{s_{\alpha}^{(2)}-s_{\alpha}^{(1)}}, \quad Q^{u}=\frac{s_{\alpha}^{(1)}+s_{\alpha}^{(2)}+u_{x}}{s_{\alpha}^{(2)}-s_{\alpha}^{(1)}} .
$$

Here $s_{\alpha}^{(1)}, s_{\alpha}^{(2)}$ are two different solutions of (11), (12) for the same parameter $\alpha$. This symmetry depends upon $\alpha$; expansion in a (formal) power series in $\alpha$ will give an infinite hierarchy of symmetries. However before we do this, we exploit the fact that a generalized symmetry of the form $X=Q^{x} \frac{\partial}{\partial x}+Q^{u} \frac{\partial}{\partial u}$ which acts on both the dependent and independent variables can be transformed to a generalized symmetry which acts only on the dependent variable [46] with characteristic $Q=Q^{u}-Q^{x} u_{x}$. Here we have

$$
Q=\frac{s_{\alpha}^{(1)}+s_{\alpha}^{(2)}}{s_{\alpha}^{(2)}-s_{\alpha}^{(1)}}
$$

This is also the characteristic for a symmetry of the full family of equations (19).

The next thing to do is to find a (formal) asymptotic series solution of (11)-(12) for small $|\alpha|$. This takes the form

$$
s_{\alpha}=\sum_{n=1}^{\infty} s_{n} \alpha^{\frac{n}{2}}
$$

where

$$
s_{1}=\sqrt{m}, \quad s_{2}=-\frac{s_{1, x}}{s_{1}}, \quad s_{n+1}=-\frac{s_{n, x}}{s_{1}}+\frac{1}{2 s_{1}}\left(\delta_{n, 2}-\sum_{i=0}^{n-2} s_{i+2} s_{n-i}\right), \quad n=2,3 \ldots
$$

A second solution of (111)-(12) can be obtained by replacing $\alpha^{\frac{1}{2}}$ by $-\alpha^{\frac{1}{2}}$. So we get

$$
s_{\alpha}^{(1)}=\sum_{n=1}^{\infty} s_{n} \alpha^{\frac{n}{2}}, \quad s_{\alpha}^{(2)}=\sum_{n=1}^{\infty} s_{n}\left(-\alpha^{\frac{1}{2}}\right)^{n} .
$$

Plugging this into (52) we obtain

$$
\frac{Q}{\sqrt{\alpha}}=\frac{\sum_{n=1}^{\infty} s_{2 n} \alpha^{n}}{\sum_{n=1}^{\infty} s_{2 n-1} \alpha^{n}}
$$

The expansion of (55) around $\alpha=0$ gives an infinite hierarchy of symmetries of $\mathrm{CH}$. The first few of these take the form

$$
\begin{aligned}
& X_{1}=\left(\frac{1}{\sqrt{m}}\right)_{x} \frac{\partial}{\partial u} \\
& X_{2}=\left(\frac{4 m m_{x x}-5 m_{x}^{2}+4 m^{2}}{m^{7 / 2}}\right)_{x} \frac{\partial}{\partial u} \\
& X_{3}=\left(\frac{-64 m^{3} m_{x x x x}-448 m^{2} m_{x} m_{x x x}-160 m^{3} m_{x x}+1848 m m_{x}^{2} m_{x x}}{m^{13 / 2}}\right)_{x} \frac{\partial}{\partial u} .
\end{aligned}
$$


The fact that all the characteristics are $x$-derivatives is indicative that these symmetries can be derived from corresponding symmetries of $\mathrm{pCH}$. The generating symmetry for $\mathrm{pCH}$ (up to an irrelevant overall constant factor) is thus $Q^{v} \frac{\partial}{\partial v}$ where

$$
Q^{v}=\frac{1}{s_{\alpha}^{(1)}-s_{\alpha}^{(2)}}
$$

As stressed before, the symmetry with characteristic (52) is a symmetry for the full family of equations (19), including the HS equation. For HS the asymptotic series solutions of (23)-(24) takes the form

$$
s_{\alpha}^{(1)}=\sum_{n=1}^{\infty} s_{n} \alpha^{\frac{n}{2}}, \quad s_{\alpha}^{(2)}=\sum_{n=1}^{\infty} s_{n}\left(-\alpha^{\frac{1}{2}}\right)^{n},
$$

where

$$
s_{1}=i \sqrt{u_{x x}}, \quad s_{2}=-\frac{s_{1, x}}{s_{1}}, \quad s_{n+1}=-\frac{s_{n, x}}{s_{1}}-\frac{1}{2 s_{1}}\left(\sum_{i=0}^{n-2} s_{i+2} s_{n-i}\right), \quad n=2,3 \ldots
$$

Proceeding as before gives an infinite hierarchy of symmetries for HS, with the first few taking the form

$$
\begin{aligned}
& X_{1}=\left(\frac{1}{\sqrt{u_{x x}}}\right)_{x} \frac{\partial}{\partial u}, \\
& X_{2}=\left(4 \frac{u_{x x x x}}{u_{x x}^{5 / 2}}-5 \frac{u_{x x x}^{2}}{u_{x x}^{7 / 2}}\right)_{x} \frac{\partial}{\partial u}, \\
& X_{3}=\left(1155 \frac{u_{x x x}^{4}}{u_{x x}^{13 / 2}}-1848 \frac{u_{x x x}^{2} u_{x x x x}}{u_{x x}^{11 / 2}}+448 \frac{u_{x x x} u_{x x x x x}}{u_{x x}^{9 / 2}}+336 \frac{u_{x x x x}^{2}}{u_{x x}^{9 / 2}}-64 \frac{u_{x x x x x x}}{u_{x x}^{7 / 2}}\right)_{x} \frac{\partial}{\partial u} .
\end{aligned}
$$

Using the fact that if a single solution of the Riccati equation (11) is known then it is possible to find the general solution by quadratures, it is possible to rewrite (52) in the form

$$
Q=1+\frac{s_{\alpha}^{(1)}(x)}{\alpha}\left(\int_{x_{0}}^{x} e^{\frac{1}{\alpha} \int_{y}^{x} s_{\alpha}^{(1)}(z) d z} d y+C e^{\frac{1}{\alpha} \int_{x_{0}}^{x} s_{\alpha}^{(1)}(z) d z}\right) .
$$

and (59) in the form

$$
Q^{v}=\int_{x_{0}}^{x} e^{\frac{1}{\alpha} \int_{y}^{x} s_{\alpha}^{(1)}(z) d z} d y+C e^{\frac{1}{\alpha} \int_{x_{0}}^{x} s_{\alpha}^{(1)}(z) d z} .
$$

Here $C$ is an arbitrary constant. Since a linear combination of symmetries is a symmetry, both terms on the RHS are by themselves the characteristics of symmetries. The symmetry associated with the factor multiplying $C$ is the nonlocal symmetry first presented in [56]. The relation between Bäcklund transformations and nonlocal symmetries has recently been discussed in 42 .

A conservation law (CL) for a PDE for the scalar function $u(x, t)$ is an expression

$$
T_{t}+X_{x}=0
$$

which holds on solutions of the equation. Conservation laws for $\mathrm{CH}$ can be obtained from (13) by writing it in the form

$$
s_{t}+\left(s u-\alpha\left(u_{x}+2 s\right)\right)_{x}=0
$$


Using the expansion (53) for $s$ in (61) we obtain an infinite hierarchy of conservation laws. Terms with integer powers of $\alpha$ in this expansion give trivial CLs. To prove this, observe from (54) that the terms with integer powers are obtained by setting $s=\frac{1}{2}\left(s_{\alpha}^{(1)}+s_{\alpha}^{(2)}\right)$ in (61). But from (11) it is simple to verify that

$$
\frac{1}{2}\left(s_{\alpha}^{(1)}+s_{\alpha}^{(2)}\right)=-\alpha\left(\ln \left|s_{\alpha}^{(1)}-s_{\alpha}^{(2)}\right|\right)_{x} .
$$

Thus to obtain nontrivial laws we look at only the half-integer powers of $\alpha$. Thus we set $s=s_{\alpha}^{(1)}-s_{\alpha}^{(2)}$ in (61) to obtain the generating conservation law

$$
\begin{aligned}
T & =s_{\alpha}^{(1)}-s_{\alpha}^{(2)} \\
X & =(u-2 \alpha)\left(s_{\alpha}^{(1)}-s_{\alpha}^{(2)}\right) .
\end{aligned}
$$

The expansion of the generating conservation law around $\alpha=0$ gives an infinite hierarchy of nontrivial CLs for $\mathrm{CH}$ and $\mathrm{pCH}$. The first few take the form

$$
\begin{aligned}
T_{1}= & \sqrt{m} \\
X_{1}= & u T_{1} \\
T_{2}= & \frac{1}{8 m^{5 / 2}}\left(4 m^{2}+4 m m_{x x}-5 m_{x}^{2}\right), \\
X_{2}= & u T_{2}-2 T_{1}, \\
T_{3}= & \frac{1}{128 m^{11 / 2}}\left(64 m^{3} m_{x x x x}-1105 m_{x}^{4}+1768 m m_{x}^{2} m_{x x}-304 m^{2} m_{x x}^{2}-448 m^{2} m_{x} m_{x x x}\right. \\
& \left.-96 m^{3} m_{x x}+200 m^{2} m_{x}^{2}-16 m^{4}\right), \\
X_{3}= & u T_{3}-2 T_{2} .
\end{aligned}
$$

Similar results can be obtained for HS and the full family of equations (19). The existence of an infinite number of conservation laws for $\mathrm{CH}$ follows from the bihamiltonian structure for $\mathrm{CH}$ discovered in [9]. The local form of these conservations laws was first obtained in [23], and they were subsequently further studied, and their derivation simplified, in numerous works such as [56, 57, 28, 25, 27, 35, 11, 30, 24. The derivation given here can be easily related to previous ones, though the use of 2 solutions of (11)-(12) to understand the triviality of "half" of the conservation laws, is, we believe, new.

\section{Concluding remarks}

In this paper we have explored the theory of the Bäcklund transformation for the CamassaHolm equation. This is an unfamiliar type of BT, as it acts on one of the independent variables, as well as the dependent variables. However, it has emerged that it is just as useful - using the superposition principles for the action on the different variables, we can exploit the BT to write down two wave solutions, just as is done for standard integrable equations such as KdV. Furthermore, we have shown how a double BT encodes an infinite set of symmetries for $\mathrm{CH}$, and the relationship of the $\mathrm{BT}$ and conservation laws.

We have seen that the BT can also generate "unphysical" solutions, by which we mean solutions for which the new independent variable is not a $1-1$ function of the old independent variable. Going beyond two wave solutions, it is not clear exactly what superpositions are allowed without creating singularities, though it seems to be a reasonable hypothesis that all possible combinations of solitons and cuspons can be formed, 
with the speeds permitted by the value of $u_{0}$, as listed in the table at the end of section 5. It seems to us that this is a problem that remains to be handled indepdendent of the method used for constructing multiwave solutions.

Peakons emerge from both solitons and cuspons in the limit $u_{0} \rightarrow 0$ (with one giving rise to peakons of positive speed and one to peakons of negative speed, depending on whether the limit is taken from below or from above). This is an extremely singular limit. We have not yet found a way to apply a superposition principle directly to peakons, but we continue to search.

Finally, one more general comment. The BT, in its minimalist form, is the transformations (10) and (14) where $s$ satisfies (11)-(12). The latter equations for $s$ are equivalent to the Lax pair, or linear system, for $\mathrm{CH}$. So the BT seems to be more than the linear system. We wonder if there is a case of an integrable system without a BT?

\section{References}

[1] Adler, V. E., Bobenko, A. I., And Suris, Y. B. Classification of integrable equations on quad-graphs. The consistency approach. Comm. Math. Phys. 233, 3 (2003), 513-543.

[2] Atkinson, J., And Nieszporski, M. Multi-quadratic quad equations: integrable cases from a factorized-discriminant hypothesis. Int. Math. Res. Not. IMRN, 15 (2014), 4215-4240.

[3] B̈̈CKLund, A. Om ytor med konstant negativ krökning. F. Berlings boktr, 1883.

[4] Beals, R., Sattinger, D. H., And Szmigielski, J. Multi-peakons and a theorem of Stieltjes. Inverse Problems 15, 1 (1999), L1-L4.

[5] Beals, R., Sattinger, D. H., And Szmigielski, J. Multipeakons and the classical moment problem. Adv. Math. 154, 2 (2000), 229-257.

[6] Beals, R., Sattinger, D. H., And Szmigielski, J. Peakon-antipeakon interaction. J. Nonlinear Math. Phys. 8, suppl. (2001), 23-27. Nonlinear evolution equations and dynamical systems (Kolimbary, 1999).

[7] Boyd, J. P. Peakons and coshoidal waves: traveling wave solutions of the CamassaHolm equation. Appl. Math. Comput. 81, 2-3 (1997), 173-187.

[8] Boyd, J. P. Near-corner waves of the Camassa-Holm equation. Phys. Lett. A 336, 4-5 (2005), 342-348.

[9] Camassa, R., And Holm, D. D. An integrable shallow water equation with peaked solitons. Phys. Rev. Lett. 71 (Sep 1993), 1661-1664.

[10] Camassa, R., Holm, D. D., and Hyman, J. M. A new integrable shallow water equation. Advances in Applied Mechanics 31, 31 (1994), 1-33.

[11] Casati, P., Lorenzoni, P., Ortenzi, G., and Pedroni, M. On the local and nonlocal Camassa-Holm hierarchies. J. Math. Phys. 46, 4 (2005), 042704, 8.

[12] Chertock, A., Liu, J.-G., And Pendleton, T. Elastic collisions among peakon solutions for the Camassa-Holm equation. Appl. Numer. Math. 93 (2015), 30-46. 
[13] Constantin, A. On the scattering problem for the Camassa-Holm equation. $R$. Soc. Lond. Proc. Ser. A Math. Phys. Eng. Sci. 457, 2008 (2001), 953-970.

[14] Constantin, A., And Lenells, J. On the inverse scattering approach for an integrable shallow water wave equation. Phys. Lett. A 308, 5-6 (2003), 432-436.

[15] Constantin, A., And Strauss, W. A. Stability of a class of solitary waves in compressible elastic rods. Phys. Lett. A 270, 3-4 (2000), 140-148.

[16] Constantin, A., And Strauss, W. A. Stability of peakons. Comm. Pure Appl. Math. 53, 5 (2000), 603-610.

[17] Constantin, A., And Strauss, W. A. Stability of the Camassa-Holm solitons. J. Nonlinear Sci. 12, 4 (2002), 415-422.

[18] DAI, H.-H., AND LI, Y. The interaction of the $\omega$-soliton and $\omega$-cuspon of the Camassa-Holm equation. J. Phys. A 38, 42 (2005), L685-L694.

[19] DAI, H.-H., LI, Y., AND Su, T. Multi-soliton and multi-cuspon solutions of a Camassa-Holm hierarchy and their interactions. J. Phys. A 42, 5 (2009), 055203, 13.

[20] Dullin, H. R., Gottwald, G. A., and Holm, D. D. An integrable shallow water equation with linear and nonlinear dispersion. Phys. Rev. Lett. 87, 19 (2001), 194501.

[21] Feng, B.-F., Maruno, K.-I., And Ohta, Y. A self-adaptive moving mesh method for the Camassa-Holm equation. J. Comput. Appl. Math. 235, 1 (2010), 229-243.

[22] Ferreira, M. C., Kraenkel, R. A., And Zenchuk, A. I. Soliton-cuspon interaction for the Camassa-Holm equation. J. Phys. A 32, 49 (1999), 8665-8670.

[23] Fisher, M., And Schiff, J. The Camassa Holm equation: conserved quantities and the initial value problem. Phys. Lett. A 259, 5 (1999), 371-376.

[24] Golovko, V., Kersten, P., Krasilshchik, I., And Verbovetsky, A. On integrability of the CamassaHolm equation and its invariants. Acta Applicandae Mathematicae 101, 1-3 (2008), 59-83.

[25] Górka, P., And Reyes, E. G. The modified Camassa-Holm equation. Int. Math. Res. Not. IMRN, 12 (2011), 2617-2649.

[26] Guo, B., And Liu, Z. Peaked wave solutions of CH-r equation. Sci. China Ser. A 46, 5 (2003), 696-709.

[27] Heredero, R. H., And Reyes, E. G. Geometric integrability of the CamassaHolm equation. ii. Internat. Math. Res. Notices 2012, 13 (2012), 3089-3125.

[28] Hernández-Heredero, R., And Reyes, E. G. Nonlocal symmetries and a Darboux transformation for the Camassa-Holm equation. J. Phys. A 42, 18 (2009).

[29] Hunter, J. K., And Zheng, Y. X. On a completely integrable nonlinear hyperbolic variational equation. Phys. D 79, 2-4 (1994), 361-386. 
[30] Ivanov, R. I. Extended Camassa-Holm hierarchy and conserved quantities. Zeitschrift für Naturforschung A 61, 3-4 (2006), 133-138.

[31] Johnson, R. S. On solutions of the Camassa-Holm equation. R. Soc. Lond. Proc. Ser. A Math. Phys. Eng. Sci. 459, 2035 (2003), 1687-1708.

[32] Kalisch, H., And Lenells, J. Numerical study of traveling-wave solutions for the Camassa-Holm equation. Chaos Solitons Fractals 25, 2 (2005), 287-298.

[33] Kraenkel, R. A., And Zenchuk, A. Camassa-Holm equation: transformation to deformed sinh-Gordon equations, cuspon and soliton solutions. J. Phys. A 32, 25 (1999), 4733-4747.

[34] LAmB, JR., G. L. Bäcklund transformations for certain nonlinear evolution equations. J. Mathematical Phys. 15 (1974), 2157-2165.

[35] Lenells, J. Conservation laws of the Camassa-Holm equation. J. Phys. A 38, 4 (2005), 869-880.

[36] Lenells, J. Stability for the periodic Camassa-Holm equation. Math. Scand. 97, 2 (2005), 188-200.

[37] Lenells, J. Traveling wave solutions of the Camassa-Holm equation. J. Differential Equations 217, 2 (2005), 393-430.

[38] LI, Y. Some water wave equations and integrability. J. Nonlinear Math. Phys. 12, suppl. 1 (2005), 466-481.

[39] Li, Y., AND Zhang, J. E. The multiple-soliton solution of the Camassa-Holm equation. Proc. R. Soc. Lond. Ser. A Math. Phys. Eng. Sci. 460, 2049 (2004), 26172627.

[40] LI, Y. A., AND Olver, P. J. Convergence of solitary-wave solutions in a perturbed bi-Hamiltonian dynamical system. I. Compactons and peakons. Discrete Contin. Dynam. Systems 3, 3 (1997), 419-432.

[41] Li, Y. A., AND Olver, P. J. Convergence of solitary-wave solutions in a perturbed bi-Hamiltonian dynamical system. II. Complex analytic behavior and convergence to non-analytic solutions. Discrete Contin. Dynam. Systems 4, 1 (1998), 159-191.

[42] Lou, S. Y., Hu, X., And Chen, Y. Nonlocal symmetries related to bcklund transformation and their applications. J. Phys. A 45, 15 (2012), 155209.

[43] Matsuno, Y. Parametric representation for the multisoliton solution of the Camassa-Holm equation. Journal of the Physical Society of Japan 74, 7 (2005), 1983-1987.

[44] Matsuno, Y. Cusp and loop soliton solutions of short-wave models for the Camassa-Holm and Degasperis-Procesi equations. Phys. Lett. A 359, 5 (2006), 451457.

[45] Ohta, Y., Maruno, K.-I., And Feng, B.-F. An integrable semi-discretization of the Camassa-Holm equation and its determinant solution. J. Phys. A 41, 35 (2008), 355205,30 . 
[46] Olver, P. J. Applications of Lie groups to Differential Equations, 2nd ed. SpringerVerlag, New York, 1993.

[47] PArker, A. On the Camassa-Holm equation and a direct method of solution. I. Bilinear form and solitary waves. Proc. R. Soc. Lond. Ser. A Math. Phys. Eng. Sci. 460, 2050 (2004), 2929-2957.

[48] Parker, A. On the Camassa-Holm equation and a direct method of solution. II. Soliton solutions. Proc. R. Soc. Lond. Ser. A Math. Phys. Eng. Sci. 461, 2063 (2005), 3611-3632.

[49] Parker, A. On the Camassa-Holm equation and a direct method of solution. III. $N$-soliton solutions. Proc. R. Soc. Lond. Ser. A Math. Phys. Eng. Sci. 461, 2064 (2005), 3893-3911.

[50] Parker, A. Cusped solitons of the Camassa-Holm equation. I. Cuspon solitary wave and antipeakon limit. Chaos Solitons Fractals 34, 3 (2007), 730-739.

[51] PARker, A. Wave dynamics for peaked solitons of the Camassa-Holm equation. Chaos Solitons Fractals 35, 2 (2008), 220-237.

[52] Parker, A. Cusped solitons of the Camassa-Holm equation. II. Binary cusponsoliton interactions. Chaos Solitons Fractals 41, 3 (2009), 1531-1549.

[53] Parker, A., And Matsuno, Y. The peakon limits of soliton solutions of the Camassa-Holm equation. Journal of the Physical Society of Japan 75, 12 (2006), 124001.

[54] Parkes, E., And Vakhnenko, V. Explicit solutions of the CamassaHolm equation. Chaos, Solitons and Fractals 26, 5 (2005), 1309 - 1316.

[55] Rasin, A. G., And Schiff, J. The Gardner method for symmetries. J. Phys. A 46, 15 (2013), 155202.

[56] Reyes, E. G. Geometric integrability of the Camassa-Holm equation. Lett. Math. Phys. 59, 2 (2002), 117-131.

[57] Reyes, E. G. The soliton content of the Camassa-Holm and Hunter-Saxton equations. In Proceedings of Institute of Mathematics of NAS of Ukraine (2002), vol. 43, pp. 201-208.

[58] Rogers, C., And Schief, W. K. Bäcklund and Darboux transformations. Cambridge Texts in Applied Mathematics. Cambridge University Press, Cambridge, 2002. Geometry and modern applications in soliton theory.

[59] Rogers, C., And Shadwick, W. F. Bäcklund transformations and their applications, vol. 161 of Mathematics in Science and Engineering. Academic Press, Inc. [Harcourt Brace Jovanovich, Publishers], New York-London, 1982.

[60] Schiff, J. The Camassa-Holm equation: a loop group approach. Phys. D 121, 1-2 (1998), 24-43. 
[61] Schiff, J., And Twito, M. Backlund transformations for the Dym and modified Dym equations. In preparation (2015).

[62] Tychynin, V. A. Non-local symmetry and generating solutions for Harry-Dymtype equations. J. Phys. A 27, 13 (1994), 4549-4556.

[63] Xia, B., Zhou, R., And Qiao, Z. Darboux transformation and multi-soliton solutions of the Camassa-Holm equation and modified Camassa-Holm equation. arXiv preprint arXiv:1506.08639 (2015).

[64] Zhang, L., Chen, L.-Q., And Huo, X. Peakons and periodic cusp wave solutions in a generalized Camassa-Holm equation. Chaos Solitons Fractals 30, 5 (2006), 12381249 . 